\title{
Telemedicine in Pediatric Sleep
}

Shalini Paruthi, MD ${ }^{a, b, *}$

\section{KEYWORDS}

$\bullet$ Pediatric • Sleep $\bullet$ Telemedicine $\bullet$ Child $\bullet$ Apnea $\bullet$ Insomnia

\section{KEY POINTS}

- There are a multitude of potential benefits to incorporating telemedicine into pediatric sleep practices, including and not limited to saving travel time and costs, fewer school absences, less missed work time for parents, and bringing multiple caregivers together conveniently (such as parents, babysitter, teachers, and grandparents), who all may provide care for a child and provide different perspectives on how the child's sleep problem is affecting overall daytime function and behavior.

- Telemedicine can be delivered synchronously or asynchronously and through many modalities, of phone, video, text, Internet Web sites, podcasts, audio recordings, social media/YouTube, and mobile applications.

- Some pediatric sleep disorders may be completely and appropriately evaluated, diagnosed, and treated during virtual video visits because the physical examination findings may add little or no additional significant information. These specifically may include circadian rhythm disorders, in particular delayed sleep-wake phase disorder, insomnia, and sleep-related movement disorders, such as restless legs syndrome.

- Other sleep disorders, such as obstructive sleep apnea, may be better evaluated in office or with a hybrid model of some in-office visits and some virtual video visits. Tonsil size can be difficult to evaluate over a virtual visit. There are unique benefits to virtual visits from home vs in-office visits for the child struggling with continuous positive airway pressure therapy.

\section{INTRODUCTION}

Telemedicine in pediatrics has become an important consideration since the coronavirus disease 2019 (COVID-19) pandemic, in an effort to reduce risk of illness to pediatric patients, their families, staff, and clinicians. Prior to the COVID-19 pandemic, few clinicians engaged in routine telemedicine visits because thorough examinations were difficult to perform; there was lack of consistent reimbursement; and many clinicians were busy with their in-office practices. This changed abruptly in March 2020, in the United States, when many states issued stay-at-home-type orders, thus effectively causing in-office visits to plummet. Many sleep centers had to close their offices or sleep laboratories temporarily.

Yet, children need sleep care. Most clinical sleep medicine practices immediately searched for effective ways to deliver telemedicine services, ranging from telephone calls to video visits. This article reviews how telemedicine has been explored in pediatric sleep medicine prior to the pandemic, current applications of telemedicine, challenges, and reimagining pediatric sleep within the realm of telemedicine.

Fortunately, most clinicians already have engaged in some type of telemedicine when calling patient families to deliver care, such as phone calls

The author has no commercial or financial conflicts of interest or funding sources for this article.

a Sleep Medicine and Research Center, St. Luke's Hospital, 232 South Woods Mill Road, Chesterfield, MO 63017, USA; ${ }^{b}$ Pediatrics, Saint Louis University School of Medicine, St Louis, MO, USA

* Sleep Medicine and Research Center, St. Luke's Hospital, 232 South Woods Mill Road, Chesterfield, MO 63017.

E-mail address: Shalini.Paruthi@stlukes-stl.com 
to review results or get more information and create a treatment plan for a specific question or problem or communicating with patients through their electronic health record systems. Additionally, many clinicians have Web sites containing education materials and videos. Many of these communication types, however, previously were not consistently billable as they are now. For pediatric care coverage, the American Academy of Pediatrics maintains a state-by-state list of telehealth policy changes during the pandemic: https:// downloads.aap.org/DOCCSA/State-TelehealthNotices.pdf.

A list of state-by-state telehealth laws, updated prior to the pandemic in Fall 2019, can be found at https://www.cchpca.org/sites/default/files/ 2019-10/50\%20State\%20Telehalth\%20Laws\% 20and\%20Reibmursement\%20Policies \% 20Report\%20Fall\%202019\%20FINAL.pdf. At the time of writing, it is unknown which coverage insurers will maintain after the pandemic subsides, and readers are directed to check these Web sites frequently for updated information as it becomes available.

There are a multitude of potential benefits to incorporating telemedicine into pediatric sleep practices, including and not limited to saving travel time and costs; fewer school absences; less missed work time for parents; and bringing multiple caregivers together conveniently, such as parents, babysitter, teachers, and grandparents, who all may provide care for a child and provide different perspectives on how the child's sleep problem is affecting overall daytime function and behavior. Telemedicine offers a variety of treatment options that are easily applied within sleep disorders care, such as delivering cognitive behavior therapy for insomnia (CBTI) and follow-up visits for continuous positive airway pressure therapy (CPAP) adherence, particularly in the early weeks or months when more frequent check-ins may improve adherence to therapy. Ubiquitous smartphones allow almost all caregivers to connect seamlessly and attend telemedicine appointments, which also may decrease appointment no-show rates.

\section{USE OF TELEMEDICINE IN PEDIATRIC SLEEP}

Telemedicine can be delivered synchronously or asynchronously and through many modalities, of phone, video, text, Internet Web sites, podcasts, audio recordings, social media/YouTube, and applications (apps). This article focuses on delivery of health care, not diagnostics or data collection; thus, tools, including home sleep apnea test devices, oximeters, capnography, actigraphs, and wearables, are not reviewed.
Examples of prepandemic telemedicine in children with sleep problems are abundant in the literature, and a sampling is reviewed to illustrate a variety of modalities that have been tested for feasibility and if outcomes were impacted. For example, in a study of mothers of healthy term newborns, mothers received 60 days of frequent e-mails or text messages containing short safe sleep videos. The investigators found this mobile health intervention improved self-reported adherence to 4 infant safe sleep practices compared with control interventions. ${ }^{1,2}$

Telemedicine through phone call follow up-visits also has been studied in pediatric obstructive sleep apnea (OSA).$^{3-5}$ Most recently, a study found that of 535 children who underwent an otolaryngologic (ear, nose, and throat) procedure of adenoidectomy, adenotonsillectomy, or tonsillectomy, all 535 parents were contacted by phone 6 weeks after the procedure to assess the need for further follow-up in the clinic; 55 of these parents were invited to evaluate the nurse-led telephone visit and $100 \%$ were "fully satisfied" and felt the phone call follow-up provided convenience, personalized care, and reassurance. In all, only 11 of the 55 children were seen later in the clinic. ${ }^{3}$

Similarly, telemedicine delivery by phone has been applied in the treatment of insomnia. A randomized controlled trial of distanced telephonecoached intervention of insomnia in 61 children with insomnia and attention-deficit/hyperactivity disorder showed reduction of sleep problems and increase in psychosocial functioning at the conclusion of the 5 phone calls time period and follow-up 6 months later. Parents had completed 5 telephone sessions, done weekly. ${ }^{6}$ The same group of investigators then applied the insomnia interventions in children with neurodevelopmental disorders, with parents noting the telephone calls were helpful and feasible. ${ }^{7}$

Published literature includes many studies on Internet-based telemedicine within pediatric sleep, particularly for treatment of insomnia in adolescents. ${ }^{8}$ In a randomized controlled trial of 116 children, ages 12 years old to 19 years old, adolescents were enrolled to receive either Internet-based CBTI or group CBTI (groups of 6-8 participants). The Internet intervention utilized videos, automated and written feedback, instruction, exercises, interactive questionnaires, and personalized bedtime advice, similar to the information provided to the in-person group. Sleep outcomes from the 2 CBTI groups were compared with sleep in adolescents on a wait list group. Children who received CBTI by Internet delivery or in-person delivery had significantly 
improved sleep parameters compared with children in the wait list group. ${ }^{9}$

Audio-recordings were provided in a study of 55 children ages 6 months to 4 years and their parents and included short, calm bedtime stories and also imaginative exercise, which could be downloaded from the Internet. Parents decreased their dysfunctional bedtime strategies and improved their limit-setting behaviors, and children had fewer nighttime wakings. ${ }^{10}$

Furthermore, podcast sessions with online blogging by caregivers were competed in a study of 34 parent-child dyads. The investigators found that the online program with a podcast and online blogging was as effective as the face-to-face program. Parent ratings improved regarding their child's bedtime resistance, sleep onset, and bedtime anxiety and decreased regarding the child's awakenings. ${ }^{11}$

Apps may be easily used by older children and adolescents. Remarkably, in a study of 88 adolescents, ages 8 years old to 17 years old, who were assessed for sleep quality and their pain from sickle cell disease and their sleep, $81.87 \%$ of possible app entries were completed. The study staff sent many reminders, contacting the children and adolescents once a week by phone and e-mail to provide feedback on compliance, provide encouragement to complete entries in the app, and problem-solve any technical or other issues. Children entered data twice a day for 4 weeks into the app on the Apple or Android device they owned or was provided to them as part of the study. They also were incentivized with gift cards for completing data entry into the apps. ${ }^{12}$

Another study in children with sickle cell looking at effects on sleep included interventions of text messages, native mobile apps, Web-based apps, mobile directly observed therapy, Internetdelivered cognitive behavior therapy, electronic pill bottle or interactive gamification (to look at monitoring of or improving medication adherence) or Android device they owned or was provided to, self-management, pain reporting and symptom reporting, stress/coping/sleep and daily activities reporting, cognitive training for memory, and guided relaxation interventions. ${ }^{13}$

Historically, interest in telemedicine began as early as the mid-1990s in sleep-related fields. As early as 1995 , otolaryngologists were exploring the idea of telemedicine for patients needing airway evaluations, some for snoring and sleep apnea, but who were located across the country. In Norway, 24 patients were examined using a video camera attached to an endoscope, by a trained general practitioner. The video was transmitted in real time to an otolaryngologist in another city in real time, who then could provide further directions and communication regarding diagnosis and treatment options. ${ }^{14}$

\section{TODAY'S TELEMEDICINE}

In general, most sleep complaints in children can be characterized broadly within difficulty falling asleep, difficulty staying asleep, abnormal behaviors in sleep, or snoring during sleep. Most of these complaints initially can be explored thoroughly through telemedicine visits. Continuity of care also can be provided for many children with chronic sleep disorders who require regular check-in visits, such as children on prescription medications for sleep or children who are on CPAP for OSA.

Telemedicine can be delivered in the form of teleconsultation in the traditional sense of caregiver and child present for a virtual video or a phone visit with sleep complaint(s) that then is explored with a clinician and treatment plan formed; teleeducation in the context of information and treatments of sleep disorders, such as cognitive behavior therapy for insomnia; and tele-research.

In the now commonplace video visit, there are several benefits of telemedicine during initial consultation. Parents can focus on the discussion with the clinician while the child is in the typical environment at home with the child's usual toys. The child can see and interact with the clinician without feeling threatened by the fear of "shots" or the "white coat." The clinician also has an opportunity to explore the child's sleep environment if the caregiver and child are at home. Conversely, sometimes the child drops out of view while playing with toys, and it can be more difficult to observe and see how the child reacts to different stimuli. The child's cooperation or hindrance may limit portions of the physical examination, such as examination of the oropharynx. For example, in the evaluation for possible OSA, tonsils can be difficult to estimate in size in 2-dimensional viewing. With the assistance of children and their parents, the author finds the clearest view of the oropharynx to be if the caregiver takes a photo on a smartphone and texts/ emails it to the clinician. If this is not possible, then in real-time video, the video from the camera on the back-side of the smartphone may be more likely to provide a clearer view of the oropharynx than the selfie view (Figs. 1-3).

Some pediatric sleep disorders may be appropriately evaluated, diagnosed, and treated completely during virtual video visits because the physical examination findings may add little or no additional significant information. These specifically include circadian rhythm disorders, in particular delayed sleep-wake phase disorder, insomnia, and sleep- 


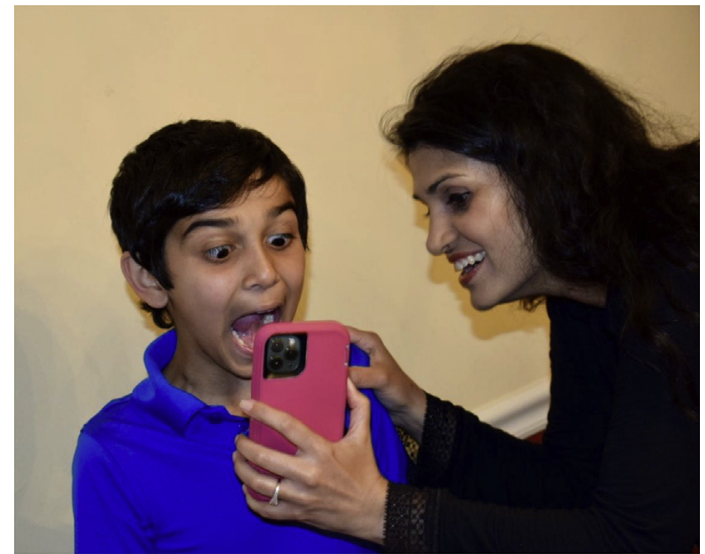

Fig. 1. Caregiver facilitating child oral examination over virtual video visit for evaluation of possible OSA.

related movement disorders, such as restless legs syndrome. During an initial visit for delayed sleepwake phase disorder, caregivers and the child often are able to articulate a through history, for which a comprehensive plan beginning with light therapy, possible melatonin use, possible chronotherapy recommendations, and behavior therapy can be developed. Virtual visits for insomnia often are discussion-heavy visits or medication follow-ups,

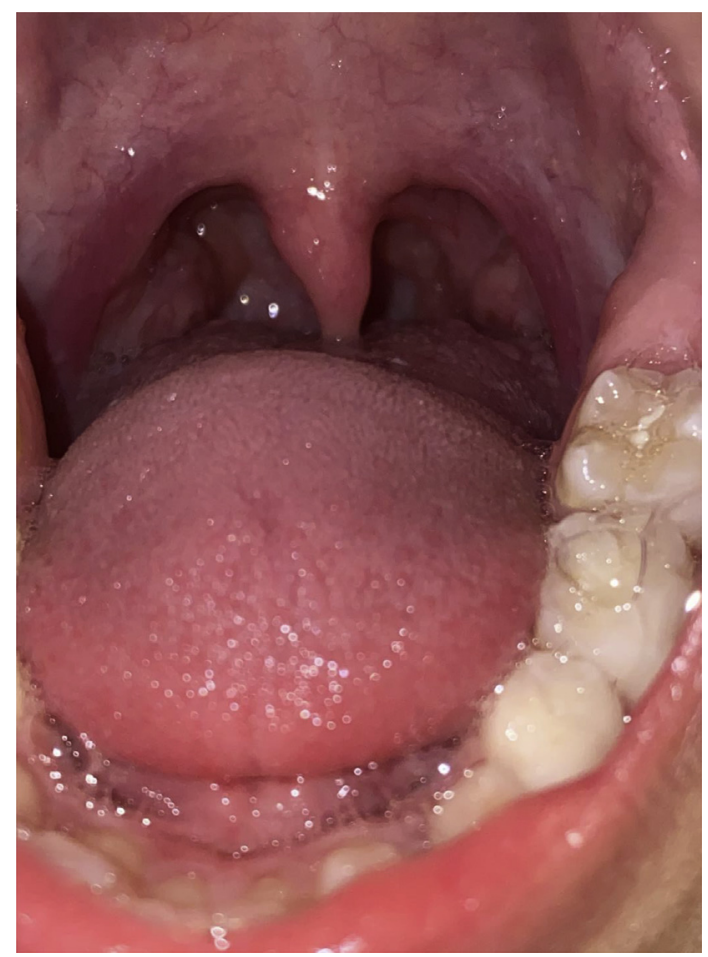

Fig. 2. Oral airway examination photo taken by parent sent by email to clinician.

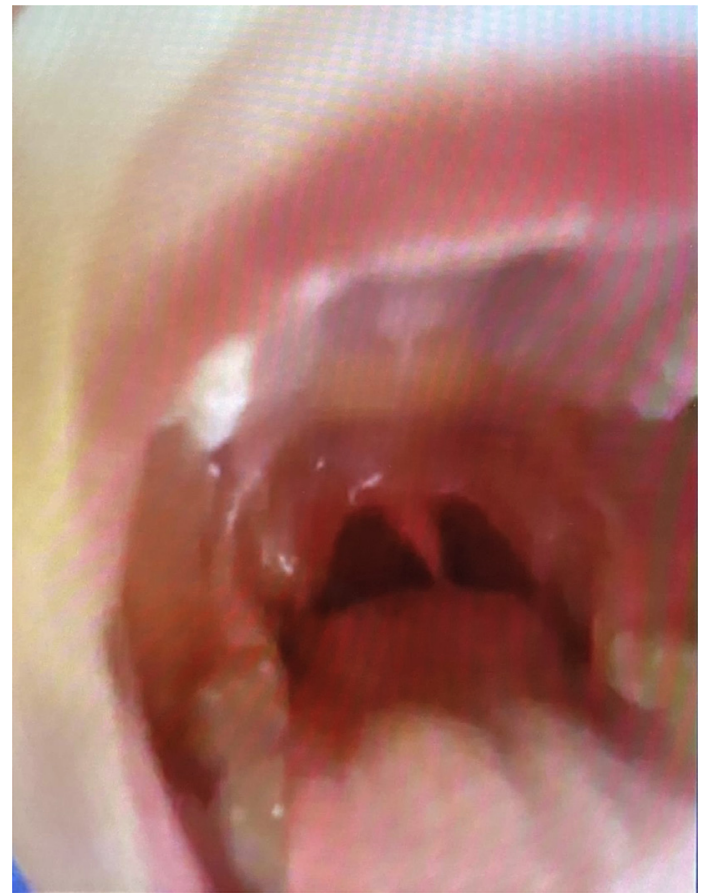

Fig. 3. Same oral airway examination as viewed onscreen through virtual video visit.

and it can be helpful to look at the child's room to see if there are any improvements that can be made in environmental sleep hygiene, such as blackout curtains. Similarly, with restless legs syndrome, child and caregiver may be able to provide a clear history, which allows evaluation to begin with testing ferritin/iron stores or other nonpharmacologic treatment strategies.

Conversely, some sleep disorders may be better evaluated in office or with a hybrid model of some in-office visits and some virtual video visits. In a diagnosis of OSA, it is helpful to assess the oropharynx thoroughly, as discussed previously, and listen to the cardiovascular and pulmonary examinations for possible right-heart sided consequence (very rare!), before proceeding to polysomnogram (PSG) or otolaryngology referral. Additionally, these in-office visits frequently include a tour of the sleep laboratory, so the child knows what to expect when returning for the sleep study. For children who go on to CPAP therapy, children who are adherent and doing well may be perfect candidates for virtual video visits intermittently through the year. For children who are struggling, however, an in-office visit may allow for time for CPAP desensitization, mask fittings, and additional face-to-face education. The case can also can be made that home virtual visits also may be exceptionally helpful in the setting of children treated with CPAP therapy who are struggling to 
use their equipment. In the home setting, children can demonstrate how they put on their mask, which often allows for instant trouble-shooting. It can be frustrating for family and clinicians when these children are seen in-office yet forget to bring their problematic mask, tubing, or machine. Furthermore, with virtual or in-office visits, during which PSG results are reviewed, clinicians can show raw data from the PSG in office or virtually by the share-screen feature to visually explain the extent and severity of the sleep-disordered breathing.

Parasomnia evaluation also may require a hybrid model, because some of the treatments include discussion of sleep hygiene and sleep duration, but the evaluation also may include evaluation for sleep apnea by PSG or further evaluation for restless legs syndrome because these 2 disorders also can trigger the parasomnias. Traditionally, many caregivers bring in recordings of their child's sleep to in-office visits; however, virtual video visits also provide caregivers the ability to share these videos through the share-screen feature.

\section{CHALLENGES}

Despite all the potential benefits of telemedicine, many clinicians did not utilize telemedicine routinely. Prior to the COVID-19 pandemic, insurance companies did not consistently reimburse telephone visits or video visits to make them financially sustainable for many office practices across the United States. In addition, state laws regarding telemedicine visits and originating sites (which often excluded the clinician's home), patient location (urban versus rural), and medically underserved areas created barriers to routine implementation and reimbursement for most practices. There may be laws prohibiting prescribing medications across state lines or to patients who have not been examined physically. Many clinical practices have full in-office patient loads, with several months' wait times, that there was no additional capacity to take on more patients through telemedicine.

Telemedicine is appropriate for many physical and mental health disorders within sleep; however, a thorough physical examination is not always is possible, particularly if a patient is at home (versus coming in to special telemedicine hubs that include equipment/tools and a technician or medical assistant who facilitates transmission of this information). A physical examination of the airway is helpful, particularly for evaluation of pediatric sleep disorders, such as snoring and possible sleep apnea. Although most children allow their parent or caregiver to show a close-up view of their airway, it not always is possible to get the best view due to camera angle, picture clarity, or a child's cooperation. New patient sleep visits involve a substantial amount of time to get to know a child's sleep routine, the caregiver's approach, the environment for sleep, the sleep complaints, and the questions needed to determine if there is a medical sleep problem or behavior sleep problem, followed by a treatment plan.

An additional challenge includes avoiding incomplete care. For example, in-office visits include nurses or medical assistants checking vital signs, which are not easily available for most virtual visits if a child has not been measured for height or weighed recently. Most homes do not have portable home oximetry. All of these are valuable pieces of information within the evaluation of possible sleep apnea, in which a clinician may encounter children with failure to thrive or children with obesity. There also are important parts of the physical examination that sometimes are missing on virtual visits, including overall appearance and optimal examinations of the nasal turbinates, Mallampati score, tonsils size, molar occlusion, heart, lungs, extremities, and gait, all of which can provide additional information within the evaluation of OSA or restless legs syndrome. Tools that the author uses in office, not commonly found at home, include stethoscopes, pulse oximetry, and otoscopes; and, otolaryngology colleagues may need laryngoscopes. Additionally, if nurses are providing education or CPAP acclimatization visits, these are easier to coordinate in the office while child and caregivers are available together as a captured audience, particularly if CPAP teaching is being done. Lastly, coordination of care is more straightforward at the physical checkout counter, where follow-up appointments or PSGs can be scheduled and paperwork, laboratory slips, educational handouts, after-visit summaries, or sleep logs can be printed and provided directly to the caregiver. During virtual visits, these seemingly simple straightforward tasks can be forgotten or take an enormous amount of coordination, phone calls, and time by staff. Parental consent often is provided by signature in office when requesting records or consenting to treatment. It is unclear if this may affect patient safety.

On occasion, even with advances in telemedicine care, some medical disorders have low rates of treatment success in office or by virtual visits. A randomized controlled trial investigated a parentfocused, Internet-based, healthy lifestyle program 
for preschool-aged children. Only $69 \%$ of parentchild dyads completed 5 or 6 modules and reported some improvements, but overall the Internet-based intervention did not help children reduce body mass index, despite receiving reading material, videos, activities, quizzes, and goal-setting exercises; receiving feedback by a dietician; and getting advice, weekly e-mails, a Facebook group to post on, and gift card incentives. ${ }^{15}$

Furthermore, because the COVID-19 pandemic created the need to advance telemedicine at lightning speed in most clinical practices, there has been inadequate time to assess clinician acceptance, child and caregiver acceptance, and training. Most institutions provided telemedicine training or training sessions can be found online through a variety of resources, such as the American Telemedicine Association and the American Academy of Pediatrics Web site section on telemedicine; and sleep-specific telemedicine information can be found on the American Academy of Sleep Medicine Web site.

State laws and regulations vary, and it is important to review these frequently because they continuously evolve, and state laws describe minimum requirements on privacy, security, encryption, resolution, connections, video compression, liability insurance, malpractice, and legal risks. These ongoing challenges require thoughtful solutions if telemedicine is to be sustainable as an equal alternative to in-office visits in the future.

\section{ONGOING RESEARCH AND THE FUTURE}

Studies are ongoing specifically within pediatric sleep medicine, including Telemedicine Delivery of Treatment for Sleep Disturbances in Young Children With Autism Spectrum Disorder, Implementing Behavioral Sleep Intervention in Urban Primary Care, and Feasibility Of Insomnia Tracking and Treatment In Inflammatory Bowel Disease (clinicaltrials.gov accessed on June 28, 2020). Moreover, a simple search on the clinicaltrials. gov Web site reveals 177 studies on telemedicine in children, ranging from "not yet recruiting" to "completed," spanning a broad variety of physical and mental health disorders.

The Telemedicine for Narcolepsy (TENAR) study will evaluate feasibility, accuracy, efficacy, and safety of telemedicine for the evaluation, diagnosis, and treatment of narcolepsy in children and adults. The TENAR study includes a crosssectional analysis to evaluate the accuracy of a live, interactive sleep assessment televisit for narcolepsy diagnosis. TENAR also is a randomized, controlled noninferiority trial of multidisciplinary care of narcolepsy through televisits versus standard care in-office follow-up visits. ${ }^{16}$

In 2020, the National Center for Advancing Translational Sciences awarded \$3.6 million to the team of investigators in the Supporting Pediatric Research on Outcomes and Utilization of Telehealth (SPROUT) Collaborative, which is a group of institutions and pediatric providers collaborating with the American Academy of Pediatrics to explore the best use of telemedicine in children (https://ncats.nih.gov/pubs/features/sprout-ctsa).

Additionally, smartphone and computer apps are in development that can provide feedback through artificial intelligence algorithms. ${ }^{17}$

In an ideal setting in pediatric sleep medicine, perhaps the future lies in a hybrid model of inoffice and telemedicine visits. Patient outcomes, patient and caregiver satisfaction, clinician and staff satisfaction, and insurance plans will dictate either the sustainability or the decline of telemedicine after the COVID-19 pandemic ends. Several sleep disorders clearly require some in-office or some in-laboratory evaluation whereas others are suitable for discussion-heavy virtual video visits.

\section{REFERENCES}

1. Moon RY, Hauck FR, Colson ER, et al. The effect of nursing quality improvement and mobile health interventions on infant sleep practices: a randomized clinical trial. JAMA 2017;318(4):351-9.

2. Moon RY, Corwin MJ, Kerr S, et al. Mediators of improved adherence to infant safe sleep using a mobile health intervention. Pediatrics 2019;143(5): e20182799.

3. Walijee H, Sood S, Markey A, et al. Is nurse-led telephone follow-up for post-operative obstructive sleep apnoea patients effective? A prospective observational study at a paediatric tertiary centre. Int J Pediatr Otorhinolaryngol 2020;129:109766.

4. Rosbe KW, Jones D, Jalisi SBM. Efficacy of postoperative follow-up telephone calls for patients who underwent adenotonsillectomy. Arch Otolaryngol Head Neck Surg 2000;126:718-22.

5. Mcvay MR, Kelley KR, Mathews DL, et al. Postoperative follow-up: is a phone call enough? J Pediatr Surg 2007;43:83-6.

6. Corkum P, Lingley-Pottie P, Davidson F, et al. Better nights/better days-distance intervention for insomnia in school-aged children with/without ADHD: a randomized controlled trial. J Pediatr Psychol 2016; 41(6):701-13.

7. Tan-MacNeill KM, Smitha IM, Weiss S, et al. An eHealth insomnia intervention for children with neurodevelopmental disorders: results of a usability study. Res Dev Disabil 2020;98:103573. 
8. McLay L, Sutherland D, Machalicek W, et al. Systematic review of telemedicine interventions for the treatment of sleep problems in children and adolescents. J Behav Educ 2020;29:222-45.

9. DeBruin EJ, Bögels SM, Oort FJ, et al. Improvements of adolescent psychopathology after insomnia treatment: results from a randomized controlled trial over 1 year. J Child Psychol Psychiatry 2018;59(5):509-22.

10. Schlarb AA, Brandhorst I. Mini-KiSS Online: an internet-based intervention program for parents of young children with sleep problems-influence on parental behavior and children's sleep. Nat Sci Sleep 2012;4:41-52.

11. Roberts CA, Smith KC, Sherman AK. Comparison of online and face-to-face parent education for children with autism and sleep problems. J Autism Dev Disord 2019;49:1410-22.

12. Valrie CR, Kilpatrick RL, Alston K, et al. Investigating the sleep-pain relationship in youth with sickle cell utilizing mhealth technology. J Pediatr Psychol 2019;44(3):323-32.

13. Badawy SM, Cronin RM, Hankins J, et al. Patientcentered ehealth interventions for children, adolescents, and adults with sickle cell disease: systematic review. J Med Internet Res 2018;20(7): e10940.

14. Pedersen S, Hartviksen G, Haga D. Teleconsultation of patients with otorhinolaryngologic conditions. A telendoscopic pilot study. Arch Otolaryngol Head Neck Surg 1994;120:133-6.

15. Hammersley ML, Okely AD, Batterham MJ, et al. An internet-based childhood obesity prevention program (Time2bHealthy) for parents of preschoolaged children: randomized controlled trial. J Med Internet Res 2019;21(2):e11964.

16. Ingravallo F, Vignatelli L, Pagotto $U$, et al. Protocols of a diagnostic study and a randomized controlled non-inferiority trial comparing televisits vs standard in-person outpatient visits for narcolepsy diagnosis and care: TElemedicine for NARcolepsy (TENAR). BMC Neurol 2020;20(1). https://doi.org/10.1186/ s12883-020-01762-9.

17. Lane ND, Lin M, Mohammod $M$, et al. BeWell: sensing sleep, physical activities and social interactions to promote wellbeing. Mobile Network Appl 2014;19(3):345-59. 